\title{
Unprecedented Alkene Stereocontrol in the Claisen Rearrangement of Cyclic bis-Allylic Esters
}

Chris McFarland, John Hutchison and Matthias C. McIntosh*

Dept. of Chemistry and Biochemistry, University of Arkansas, Fayetteville, AR 72701

\section{Contents}

S2-S6 Experimental procedures and characterization data

S6 References

S7-S20 $\quad{ }^{1} \mathrm{H}-$ and ${ }^{13} \mathrm{C}-\mathrm{NMR}$ data for dienes cis-2b, trans-2c, 5, 8, trans-11a, trans-11b, cis/trans-11c 
Isobutyrate ester $1 \mathrm{~b}: n \mathrm{BuLi}(6.75 \mathrm{~mL}, 1.6 \mathrm{M}$ in hexanes, $10.8 \mathrm{mmol})$ was added to a solution of the corresponding alcohol ${ }^{1}(1.53 \mathrm{~g}, 7.2 \mathrm{mmol})$ in THF $(100 \mathrm{~mL})$ at $-78{ }^{\circ} \mathrm{C}$. After addition of isobutyric anhydride $(2.4 \mathrm{~mL}, 14.4 \mathrm{mmol})$, the reaction mixture was allowed to stir at $-78^{\circ} \mathrm{C}$ for $10 \mathrm{~min}$ after which the cooling bath was removed. After $4 \mathrm{~h}$ the reaction mixture was poured in saturated $\mathrm{NaHCO}_{3}$ solution. The crude product was isolated by extractive work-up with $\mathrm{Et}_{2} \mathrm{O}$. The product was isolated without further purification to yield ester $1 \mathbf{b}$ as a clear oil $\left(2.00 \mathrm{~g}, 98 \%\right.$ yield). IR (film) $2971,1741 \mathrm{~cm}^{-}$ ${ }^{1} ;{ }^{1} \mathrm{H} \mathrm{NMR}\left(270 \mathrm{MHz}, \mathrm{CDCl}_{3}\right) \delta 1.19(\mathrm{~d}, 6 \mathrm{H}, \mathrm{J}=6.9 \mathrm{~Hz}), 1.71(\mathrm{~s}, 6 \mathrm{H}), 2.2(\mathrm{~m}, 2 \mathrm{H}), 2.6$ (heptet, 1H, J=7.1 Hz), 3.2 (sextet, 1H, J=6.1 Hz), 4.0 (d, 1H, J=11.9 Hz), 5.26 (dd, 2H, $\mathrm{J}=6.9 \mathrm{~Hz}, 17.6 \mathrm{~Hz}), 5.71(\mathrm{~m}, 1 \mathrm{H}), 5.79(\mathrm{dd}, 1 \mathrm{H}, \mathrm{J}=10.9 \mathrm{~Hz}, 17.2 \mathrm{~Hz}) ;{ }^{13} \mathrm{C}$ NMR $(67 \mathrm{MHz}$, $\left.\mathrm{CDCl}_{3}\right) \delta 18.3,19.2,19.6,31.1,35.0,45.4,67.8,82.1,113.8,115.3,127.1,132.1,139.1$, 145.4, 175.1; Anal Calcd for $\mathrm{C}_{16} \mathrm{H}_{23} \mathrm{ClO}_{2}$ : C, 67.95; H, 8.20; found: C, 67.81; $\mathrm{H}, 8.40$; MS m/z 246, 156, 43.

Isobutyrate ester 1c: Vinyl $\mathrm{MgBr}(21.2 \mathrm{~mL}, 0.9 \mathrm{M}$ in THF, $19.1 \mathrm{mmol})$ was added to a solution of trans 6-methylcarvone ${ }^{2}(2.09 \mathrm{~g}, 12.7 \mathrm{mmol})$ in $\mathrm{THF}(100 \mathrm{~mL})$ at $0{ }^{\circ} \mathrm{C}$ over 15 $\mathrm{min}$, followed by isobutyric anhydride $(722 \mu \mathrm{L}, 4.36 \mathrm{mmol})$. After warming to rt over 1 $\mathrm{h}$, the mixture was then poured into saturated $\mathrm{NaHCO}_{3}$ solution and the crude product isolated by extractive work-up with ether. The residue was purified by flash chromatography on silica gel using 10/90 ethyl acetate/hexanes to give the a 3:1 mixture of diastereomeric alcohols. The minor ( $\alpha$-hydroxy) isomer was isolated as a yellowish oil (598.6 mg, 24\%). IR (film) 3445, $2969 \mathrm{~cm}^{-1} ;{ }^{1} \mathrm{H}$ NMR (270 MHz, $\left.\mathrm{CDCl}_{3}\right) \delta 0.77$ (d, $3 \mathrm{H}, \mathrm{J}=6.9 \mathrm{~Hz}), 1.54(\mathrm{~s}, 2 \mathrm{H}), 1.63(\mathrm{~m}, 6 \mathrm{H}) ; 2.10(\mathrm{~m}, 3 \mathrm{H}), 4.74(\mathrm{~d}, 1 \mathrm{H}, \mathrm{J}=1.4 \mathrm{~Hz}), 4.77$ (s, $1 \mathrm{H}), 5.22(\mathrm{dd}, 1 \mathrm{H}, \mathrm{J}=1.5 \mathrm{~Hz}, 10.7 \mathrm{~Hz}), 5.31(\mathrm{dd}, 1 \mathrm{H}, \mathrm{J}=1.5,17.3 \mathrm{~Hz}), 5.61(\mathrm{dd}, 2 \mathrm{H}$, $\mathrm{J}=10.7 \mathrm{~Hz}, 17.3 \mathrm{~Hz}) ;{ }^{13} \mathrm{C} \mathrm{NMR}\left(67 \mathrm{MHz}, \mathrm{CDCl}_{3}\right) \delta 11.9,18.4,19.1,31.3,40.0,44.2$, $77.3,112.4,113.8,125.7,135.3,143.2,147.4$.

Vinyl $\mathrm{MgBr}(3.6 \mathrm{~mL}, 0.9 \mathrm{M}$ in THF, $3.27 \mathrm{mmol})$ was added to a solution of the alcohol (420.2 $\mathrm{mg}, 2.18 \mathrm{mmol})$ in THF $(25 \mathrm{~mL})$ at $-78^{\circ} \mathrm{C}$. After $5 \mathrm{~min}$ at $-78^{\circ} \mathrm{C}$, isobutyric anhydride $(722 \mu \mathrm{L}, 4.36 \mathrm{mmol})$ was added. The reaction mixture was allowed to stir at $78^{\circ} \mathrm{C}$ for $10 \mathrm{~min}$, after which the cooling bath was removed. After $1 \mathrm{~h}$ the mixture was poured into saturated $\mathrm{NaHCO}_{3}$ solution and the crude product isolated by extractive work-up with $\mathrm{Et}_{2} \mathrm{O}$. The product was purified by flash chromatography on silica gel using 10/90 ethyl acetate/hexanes to give ester 1c as a colorless oil (437.3 mg, 76\%). IR (film) 2970, $1736 \mathrm{~cm}^{-1}$; ${ }^{1} \mathrm{H}$ NMR $\left(270 \mathrm{MHz}, \mathrm{CDCl}_{3}\right) \delta 0.77$ (d, 3H, J=6.9 Hz), 1.17 (d, $6 \mathrm{H}, \mathrm{J}=6.9 \mathrm{~Hz}), 1.56(\mathrm{~d}, 3 \mathrm{H}, \mathrm{J}=1.4 \mathrm{~Hz}), 1.60(\mathrm{~s}, 3 \mathrm{H}), 1.70(\mathrm{~m}, 1 \mathrm{H}), 2.03(\mathrm{~m}, 2 \mathrm{H}), 2.56$ (heptet, 1H, J=6.9 Hz), $2.80(\mathrm{~m}, 1 \mathrm{H}), 4.72(\mathrm{~s}, 1 \mathrm{H}), 4.76(\mathrm{~s}, 1 \mathrm{H}), 5.13(\mathrm{dd}, 1 \mathrm{H}, \mathrm{J}=1.6 \mathrm{~Hz}$, $10.6 \mathrm{~Hz}), 5.28$ (dd, 1H, J=1.6 Hz, 17.1 Hz), 5.54 (dd, 1H, J=10.6 Hz, $17.1 \mathrm{~Hz}), 5.71$ (m, $1 \mathrm{H}) ;{ }^{13} \mathrm{C} \mathrm{NMR}\left(67 \mathrm{MHz}, \mathrm{CDCl}_{3}\right) \delta 12.1,18.4,18.7,19.3,19.4,31.2,35.0,41.8,43.7$, 84.0, 112.3, 113.1, 126.8, 132.1, 141.9, 147.6, 175.1.

Acid cis-2b: $n \mathrm{BuLi}(3.75 \mathrm{~mL}, 1.6 \mathrm{M}$ in hexanes, $6.0 \mathrm{mmol})$ was added to a solution of $\mathrm{HNiPr}_{2}(840 \mu \mathrm{L}, 6.0 \mathrm{mmol})$ in THF $(10 \mathrm{~mL})$ at $-78^{\circ} \mathrm{C}$. After $10 \mathrm{~min}$, ester $\mathbf{1 b}(572 \mathrm{mg}$, $2.0 \mathrm{mmol})$ in THF $(10 \mathrm{~mL})$ was added, followed by TMSCl $(760 \mu \mathrm{L}, 6.0 \mathrm{mmol})$. After $30 \mathrm{~min}$, the reaction mixture was poured into $1 \mathrm{~N} \mathrm{HCl}$ and the crude product isolated by extractive work-up. The product was purified by flash chromatography on silica gel 
using $\mathrm{NEt}_{3} / \mathrm{Et}_{2} \mathrm{O}$ followed by $1 \%$ acetic acid in $\mathrm{Et}_{2} \mathrm{O}$ to give acid $\mathbf{2 b}$ as an oil $(544.2 \mathrm{mg}$, $76.6 \%$ yield). IR (film) 2970, $1700 \mathrm{~cm}^{-1} ;{ }^{1} \mathrm{H} \mathrm{NMR}\left(270 \mathrm{MHz}, \mathrm{CDCl}_{3}\right) \delta 1.21(\mathrm{~s}, 3 \mathrm{H})$, $1.24(\mathrm{~s}, 3 \mathrm{H}), 1.72(\mathrm{~s}, 3 \mathrm{H}), 2.03(\mathrm{~d}, 3 \mathrm{H}, \mathrm{J}=1.2 \mathrm{~Hz}), 2.27(\mathrm{~m}, 2 \mathrm{H}), 2.70(\mathrm{~m}, 4 \mathrm{H}), 4.58(\mathrm{~d}$, $1 \mathrm{H}, \mathrm{J}=3.3 \mathrm{~Hz}), 4.76(\mathrm{~s}, 1 \mathrm{H}), 4.81(\mathrm{t}, 1 \mathrm{H}, \mathrm{J}=1.5 \mathrm{~Hz}), 5.43(\mathrm{t}, 1 \mathrm{H}, \mathrm{J}=7.2), 5.63(\mathrm{~m}, 1 \mathrm{H}) ;{ }^{13} \mathrm{C}$ NMR $\left(67 \mathrm{MHz}, \mathrm{CDCl}_{3}\right) \delta$ 21.2, 24.0, 24.6, 24.8, 26.8, 39.1, 42.6, 47.0, 67.2, 112.4, $127.2,127.8,129.2,135.6,144.6,184.1$.

Acid trans-2c: Ester 1c (91.7 mg, $0.35 \mathrm{mmol})$ was added to a solution of KHMDS (2.1 $\mathrm{mL}, 0.5 \mathrm{M}$ in toluene, $1.05 \mathrm{mmol})$ and TIPSOTf $(280 \mu \mathrm{L}, 1.05 \mathrm{mmol})$ in $\mathrm{Et}_{2} \mathrm{O}(1 \mathrm{~mL})$ at $78^{\circ} \mathrm{C}$. The reaction mixture was allowed to warm slowly to room temperature over 2-3 $\mathrm{h}$, then was poured into saturated $\mathrm{NaHCO}_{3}$ solution and the crude product isolated by extractive work-up with $\mathrm{Et}_{2} \mathrm{O}$. The residue was immediately taken up in $\mathrm{CDCl}_{3}(1 \mathrm{~mL})$ and a catalytic amount of $\mathrm{Ph}_{2} \mathrm{~S}_{2}$ added. The solution was exposed to ambient light for $1 \mathrm{~h}$ (the progress of the reaction was monitored by ${ }^{1} \mathrm{H}-\mathrm{NMR}$ analysis). The reaction mixture was concentrated in vacuo and taken up in $5 \% \mathrm{HF}$ in $\mathrm{CH}_{3} \mathrm{CN}(1 \mathrm{~mL})$. After $30 \mathrm{~min}$ the reaction mixture was concentrated in vacuo, taken up in $\mathrm{Et}_{2} \mathrm{O}$ and then poured into saturated $\mathrm{NaHCO}_{3}$ solution. The crude product isolated by extractive work-up with $\mathrm{Et}_{2} \mathrm{O}$. The product was purified by flash chromatography on silica gel using $\mathrm{NEt}_{3} / \mathrm{Et}_{2} \mathrm{O}$ followed by $1 \%$ acetic acid in $\mathrm{Et}_{2} \mathrm{O}$ to afford acid trans-2c as an oil $(39.5 \mathrm{mg}, 43 \%$ yield); IR (film) 2967, $1702 \mathrm{~cm}^{-1} ;{ }^{1} \mathrm{H}$ NMR (270 MHz, $\left.\mathrm{CDCl}_{3}\right) \delta 1.01$ (d, 3H, J=7.1 Hz), $1.19(\mathrm{~d}, 6 \mathrm{H}, \mathrm{J}=2.4 \mathrm{~Hz}), 1.66(\mathrm{~s}, 3 \mathrm{H}), 1.73(\mathrm{~s}, 3 \mathrm{H}), 2.10(\mathrm{~m}, 2 \mathrm{H}), 2.40(\mathrm{~m}, 2 \mathrm{H}), 2.87$ (q, $1 \mathrm{H}, \mathrm{J}=7.1 \mathrm{~Hz}), 3.07$ (q, 1H, J=7.3), $4.62(\mathrm{~s}, 2 \mathrm{H}), 5.33(\mathrm{t}, 1 \mathrm{H}, \mathrm{J}=7.3 \mathrm{~Hz}), 5.44(\mathrm{~m}, 1 \mathrm{H}) ;{ }^{13} \mathrm{C}$ NMR $\left(67 \mathrm{MHz}, \mathrm{CDCl}_{3}\right) \delta 8.6,20.3,20.6,22.5,24.8,25.2,25.5,32.5,37.7,44.8,109.6$, $119.6,122.8,131.4,140.9,148.5,183.9$.

Propionate ester 3: $t \mathrm{BuLi}(2.1 \mathrm{~mL}, 1.7 \mathrm{M}$ in hexanes, $3.6 \mathrm{mmol})$ was added to a solution of trans-propenyl bromide $(155 \mu \mathrm{L}, 1.8 \mathrm{mmol})$ in $\mathrm{THF}(10 \mathrm{~mL})$ at $-78^{\circ} \mathrm{C}$. After $20 \mathrm{~min}$, a solution of trans-chlorocarvone ${ }^{1}(203.6 \mathrm{mg}, 1.2 \mathrm{mmol})$ in THF $(10 \mathrm{~mL})$ was added. The reaction mixture was allowed to stir at $-78^{\circ} \mathrm{C}$ for $1 \mathrm{~h}$, then was poured into saturated $\mathrm{NaHCO}_{3}$ solution. The crude product isolated by extractive work-up with $\mathrm{Et}_{2} \mathrm{O}$. The residue was purified on silica gel using 10/90 ethyl acetate/hexanes to yield the resulting alcohol as a clear oil $\left(149.3 \mathrm{mg}, 59 \%\right.$ yield). IR (film) $3551 \mathrm{~cm}^{-1} ;{ }^{1} \mathrm{H} \mathrm{NMR}$ $\left(270 \mathrm{MHz}, \mathrm{CDCl}_{3}\right) \delta 1.64(\mathrm{q}, 3 \mathrm{H}, \mathrm{J}=2.0 \mathrm{~Hz}) ; 1.73(\mathrm{~s}, 3 \mathrm{H}) ; 1.76(\mathrm{dd}, 2 \mathrm{H}, \mathrm{J}=1.6 \mathrm{~Hz}, 7.3$ $\mathrm{Hz}) ; 2.10$ (m, 2H); 2.35 (s, 1H); 2.88 (m, 1H); 4.00 (d, 1H, J=12.3 Hz); 4.84 (s, 2H); 5.33 $(\mathrm{dq}, 1 \mathrm{H}, \mathrm{J}=1.8 \mathrm{~Hz}, 16.1 \mathrm{~Hz}) ; 5.59(\mathrm{~m}, 1 \mathrm{H}) ; 5.83(\mathrm{dq}, 1 \mathrm{H}, \mathrm{J}=6.5 \mathrm{~Hz}, 15.4 \mathrm{~Hz}) ;{ }^{13} \mathrm{C}$ NMR $\left(67 \mathrm{MHz}, \mathrm{CDCl}_{3}\right) \delta$ 17.6, 18.2, 19.6, 31.9, 45.1, 70.7, 74.8, 113.7, 124.9, 126.2, 134.6, 134.7, 145.4; Anal Calcd for $\mathrm{C}_{13} \mathrm{H}_{19} \mathrm{ClO}$ : C, 68.86; H, 8.45; Cl, 15.64; Found: C, 69.02; $\mathrm{H}, 8.45 ; \mathrm{Cl}, 15.81$.

$n \operatorname{BuLi}(2.3 \mathrm{~mL}, 2.87 \mathrm{M}$ in hexanes, $6.6 \mathrm{mmol})$ was added to a solution of the propenyl alcohol (1.0043 g, $4.4 \mathrm{mmol})$ in THF $(30 \mathrm{~mL})$ at $-78^{\circ} \mathrm{C}$. After $5 \mathrm{~min}$, propionic anhydride $(1.1 \mathrm{~mL}, 8.8 \mathrm{mmol})$ was added. The reaction mixture was allowed to stir at $78^{\circ} \mathrm{C}$ for $10 \mathrm{~min}$, then allowed to warm slowly to $\mathrm{rt}$ over the course of $1 \mathrm{~h}$. The reaction mixture was poured into saturated $\mathrm{NaHCO}_{3}$ and the crude product isolated by extractive work-up with $\mathrm{Et}_{2} \mathrm{O}$. The residue was purified on silica gel using 10/90 ethyl acetate/hexanes to yield ester 3 a clear oil (270.2 mg, $22 \%$ yield). IR (film) $1736 \mathrm{~cm}^{-1}$; 
${ }^{1} \mathrm{H}$ NMR $\left(270 \mathrm{MHz}, \mathrm{CDCl}_{3}\right) \delta 1.13(\mathrm{t}, 3 \mathrm{H}, \mathrm{J}=7.5) ; 1.68(\mathrm{~d}, 3 \mathrm{H}, \mathrm{J}=2.1 \mathrm{~Hz}) ; 1.71(\mathrm{~s}, 3 \mathrm{H})$; $1.73(\mathrm{dd}, 2 \mathrm{H}, \mathrm{J}=1.6 \mathrm{~Hz}, 6.5 \mathrm{~Hz}) ; 2.20(\mathrm{bm}, 2 \mathrm{H}) ; 2.32(\mathrm{q}, 2 \mathrm{H}, \mathrm{J}=7.5 \mathrm{~Hz}) ; 3.20(\mathrm{~m}, 1 \mathrm{H})$; $4.05(\mathrm{~d}, 1 \mathrm{H}, \mathrm{J}=11.5 \mathrm{~Hz}) ; 4.82(\mathrm{~s}, 2 \mathrm{H}) ; 5.60(\mathrm{dd}, 1 \mathrm{H}, \mathrm{J}=1.4 \mathrm{~Hz}, 15.4 \mathrm{~Hz}) ; 5.66(\mathrm{~m}, 2 \mathrm{H})$; ${ }^{13} \mathrm{C}$ NMR $\left(67 \mathrm{MHz}, \mathrm{CDCl}_{3}\right) \delta$ 9.3, 17.8, 18.5, 19.7, 28.4, 30.9, 45.6, 62.2, 82.2, 113.5, $126.4,126.5,131.8,132.8,145.5,172.7$.

TIPS ester cis-5: Isobutyrate ester $3(270.2 \mathrm{mg}, 0.96 \mathrm{mmol})$ was added to a solution of KHMDS (5.76 mL, $0.5 \mathrm{M}$ in toluene, $2.88 \mathrm{mmol})$ and TIPSOTf $(775 \mu \mathrm{L}, 2.88 \mathrm{mmol})$ in Et2O $(15 \mathrm{~mL})$ at $-78{ }^{\circ} \mathrm{C}$. The reaction mixture was allowed warm to $\mathrm{rt}$ over $2-3 \mathrm{~h}$. The mixture was then poured into saturated $\mathrm{NaHCO}_{3}$ solution and the crude product isolated by extractive work-up with $\mathrm{Et}_{2} \mathrm{O}$. The residue was purified by flash chromatography on silica gel using 1/99 ethyl acetate/hexanes to yield TIPS ester 5 as a clear oil. (379.7 $\mathrm{mg}$, $95 \%$ yield); IR (film) 2945, 1714; ${ }^{1} \mathrm{H}$ NMR $\left(270 \mathrm{MHz}, \mathrm{CDCl}_{3}\right) \delta 0.96$ (d, 3H, $\left.6.7 \mathrm{~Hz}\right)$, $1.06(\mathrm{~d}, 21 \mathrm{H}, \mathrm{J}=7.3 \mathrm{~Hz}), 1.19(\mathrm{~d}, 3 \mathrm{H}, \mathrm{J}=7.1 \mathrm{~Hz}), 1.30(\mathrm{~m}, 2 \mathrm{H}), 1.68(\mathrm{~s}, 3 \mathrm{H}), 2.02(\mathrm{~s}, 3 \mathrm{H})$, $2.21(\mathrm{~m}, 2 \mathrm{H}), 2.68(\mathrm{~m}, 2 \mathrm{H}), 3.07(\mathrm{~m}, 1 \mathrm{H}), 4.51(\mathrm{~d}, 1 \mathrm{H}, \mathrm{J}=3.4 \mathrm{~Hz}), 4.74(\mathrm{~d}, 2 \mathrm{H}, \mathrm{J}=12.9$ $\mathrm{Hz}), 5.21(\mathrm{~d}, 1 \mathrm{H}, \mathrm{J}=10.7 \mathrm{~Hz}), 5.59(\mathrm{~m}, 1 \mathrm{H}) ;{ }^{13} \mathrm{C} \mathrm{NMR}\left(67 \mathrm{MHz}, \mathrm{CDCl}_{3}\right) \delta$ 12.0, 17.1, $17.8,20.1,21.3,24.1,27.0,36.1,47.3,48.5,67.6,112.4,127.7,129.3,133.5,135.7$, 145.1, 175.8; Anal Calcd for $\mathrm{C}_{25} \mathrm{H}_{43} \mathrm{ClO}_{2} \mathrm{Si}: \mathrm{C}, 68.38 ; \mathrm{H}, 9.87$. Found: $\mathrm{C}, 68.15 ; \mathrm{H}, 10.04$.

Isobutyrate ester 6: $n \mathrm{BuLi}(2.53 \mathrm{~mL}, 1.6 \mathrm{M}$ in hexanes, $4.05 \mathrm{mmol})$ was added to a solution of the corresponding alcohol ${ }^{1}(566.1 \mathrm{mg}, 2.7 \mathrm{mmol})$ in THF $(30 \mathrm{~mL})$ at $-78{ }^{\circ} \mathrm{C}$, followed after $5 \mathrm{~min}$ by isobutyric anhydride $(901 \mu \mathrm{L}, 5.4 \mathrm{mmol})$. The reaction mixture was allowed to stir at $-78^{\circ} \mathrm{C}$ for $10 \mathrm{~min}$, then warmed to rt over $1 \mathrm{~h}$. The reaction mixture was poured in saturated $\mathrm{NaHCO}_{3}$ solution and the crude product isolated by extractive work-up with $\mathrm{Et}_{2} \mathrm{O}$. The residue was purified on silica gel using 10/90 ethyl acetate/hexanes to yield ester $\mathbf{6}$ as a clear oil $\left(745.4 \mathrm{mg}, 98 \%\right.$ ). IR (film) $2972,1735 \mathrm{~cm}^{-}$ ${ }^{1} ;{ }^{1} \mathrm{H}$ NMR $\left(270 \mathrm{MHz}, \mathrm{CDCl}_{3}\right) \delta 1.17$ (dd, 6H, J=1.6 Hz, 6.9 Hz), $1.73(\mathrm{~m}, 3 \mathrm{H}), 1.76$ (s, $3 \mathrm{H}), 2.00(\mathrm{~m}, 1 \mathrm{H}), 2.37(\mathrm{~m}, 1 \mathrm{H}), 2.60(\mathrm{~m}, 2 \mathrm{H}), 4.79(\mathrm{~s}, 1 \mathrm{H}), 4.92(\mathrm{q}, 1 \mathrm{H}, \mathrm{J}=1.4 \mathrm{~Hz}), 5.21$ (dd, $1 \mathrm{H}, \mathrm{J}=1.0 \mathrm{~Hz}, 17.4 \mathrm{~Hz}), 5.28(\mathrm{~s}, 1 \mathrm{H}), 5.38(\mathrm{dd}, 1 \mathrm{H}, \mathrm{J}=1.0 \mathrm{~Hz}, 11.2 \mathrm{~Hz}), 5.73(\mathrm{~m}$, $1 \mathrm{H}), 6.44(\mathrm{dd}, 1 \mathrm{H}, \mathrm{J}=10.7 \mathrm{~Hz}, 17.4 \mathrm{~Hz}) ;{ }^{13} \mathrm{C} \mathrm{NMR}\left(67 \mathrm{MHz}, \mathrm{CDCl}_{3}\right) \delta$ 18.7, 18.9, 19.0, $21.7,25.1,35.1,40.9,61.9,86.5,112.1,120.0,125.9,130.0,135.1,143.9,176.1$; Anal Calcd for $\mathrm{C}_{16} \mathrm{H}_{23} \mathrm{O}_{2} \mathrm{Cl}$ : C, 67.95; H, 8.20. Found: C, 67.66; H, 8.26.

Acid trans-8: Isobutyrate ester $6(307.2 \mathrm{mg}, 1.09 \mathrm{mmol})$ was added to a solution of KHMDS (6.5 mL, $0.5 \mathrm{M}$ in toluene, $3.27 \mathrm{mmol})$ and TIPSOTf ( $880 \mu \mathrm{L}, 3.27 \mathrm{mmol})$ in $\mathrm{Et}_{2} \mathrm{O}(20 \mathrm{~mL})$ at $-78^{\circ} \mathrm{C}$. The reaction mixture was allowed to war to $\mathrm{rt}$ over $2-3 \mathrm{~h}$. The mixture was then poured into saturated $\mathrm{NaHCO}_{3}$ solution and the crude product isolated by extractive work-up with $\mathrm{Et}_{2} \mathrm{O}$. The residue was immediately taken up in $5 \% \mathrm{HF}$ in $\mathrm{CH}_{3} \mathrm{CN}(25 \mathrm{~mL})$. After $30 \mathrm{~min}$ the reaction mixture was concentrated in vacuo, taken up in $\mathrm{Et}_{2} \mathrm{O}(20 \mathrm{~mL})$ and then poured into saturated $\mathrm{NaHCO}_{3}$ solution. The residue was isolated by extractive work-up with $\mathrm{Et}_{2} \mathrm{O}$ and purified by flash chromatography on silica gel using $\mathrm{NEt}_{3} / \mathrm{Et}_{2} \mathrm{O}$ followed by $1 \%$ acetic acid in $\mathrm{Et}_{2} \mathrm{O}$ to give acid $\mathbf{8}$ as an oil (157.1 mg, $51 \%$ yield); IR (film) 2972, $1701 \mathrm{~cm}^{-1} ;{ }^{1} \mathrm{H}$ NMR $\left(270 \mathrm{MHz}, \mathrm{CDCl}_{3}\right) \delta 1.26$ (d, $6 \mathrm{H}$, $\mathrm{J}=3.2 \mathrm{~Hz}), 1.80(\mathrm{~d}, 6 \mathrm{H}, \mathrm{J}=8.3 \mathrm{~Hz}), 2.10(\mathrm{~m}, 1 \mathrm{H}), 2.34(\mathrm{~m}, 2 \mathrm{H}), 2.51(\mathrm{~m}, 1 \mathrm{H}), 2.68(\mathrm{dd}$, $1 \mathrm{H}, \mathrm{J}=9.1 \mathrm{~Hz}, 14.7 \mathrm{~Hz}), 4.83(\mathrm{~s}, 1 \mathrm{H}), 4.95(\mathrm{~s}, 1 \mathrm{H}), 5.17(\mathrm{~s}, 1 \mathrm{H}), 5.51(\mathrm{t}, 1 \mathrm{H}, \mathrm{J}=8.5 \mathrm{~Hz})$, 
$5.71(\mathrm{~d}, 1 \mathrm{H}, \mathrm{J}=5.7 \mathrm{~Hz}) ;{ }^{13} \mathrm{C}$ NMR $\left(67 \mathrm{MHz}, \mathrm{CDCl}_{3}\right) \delta$ 19.9, 21.8, 24.1, 25.3, 25.9, 37.6, $42.8,45.6,56.9,112.2,122.8,126.1,129.8,139.0,144.6,184.4$.

Propionate Ester 9a: $t$-BuLi $(29.8 \mathrm{~mL}, 1.7 \mathrm{M}$ in pentane, $50.66 \mathrm{mmol})$ was added drop wise to a solution of 2-bromopropene $(2.25 \mathrm{~mL}, 25.29 \mathrm{mmol})$ in ether $(150 \mathrm{~mL})$ at -78 ${ }^{\circ} \mathrm{C}$. After $15 \mathrm{~min}$, 2-bromo-2-cyclohexen-1-one $(3.0 \mathrm{~g}, 17.2 \mathrm{mmol})$ in ether $(50 \mathrm{~mL})$ was added. After $1 \mathrm{~h}$ at $-78{ }^{\circ} \mathrm{C}$, propionic anhydride $(4.40 \mathrm{~mL}, 34.32 \mathrm{mmol})$ was added. After warming to rt, the resulting mixture was poured into saturated $\mathrm{NaHCO}_{3}$ solution and isolated by extractive workup using $\mathrm{Et}_{2} \mathrm{O}$. The crude material was purified by flash chromatography on silica gel with 10/90 ethyl acetate/hexane to give propionate $9 \mathbf{a}$ as a clear oil (3.08 g, 66\%): IR (film) 2939, 1742, $1167 \mathrm{~cm}^{-1} ;{ }^{1} \mathrm{H}$ NMR $\left(270 \mathrm{MHz}, \mathrm{CDCl}_{3}\right)$ $1.14(\mathrm{t}, \mathrm{J}=7.5 \mathrm{~Hz}, 3 \mathrm{H}), 1.61-1.76(\mathrm{~m}, 2 \mathrm{H}), 1.83(\mathrm{~s}, 3 \mathrm{H}), 1.87-1.95(\mathrm{dt}, \mathrm{J}=3.8,14.5 \mathrm{~Hz}$, $1 \mathrm{H}), 1.99-2.08(\mathrm{~m}, 1 \mathrm{H}), 2.18-2.29(\mathrm{~m}, 1 \mathrm{H}), 2.37(\mathrm{dq}, \mathrm{J}=4.8,7.5 \mathrm{~Hz}, 2 \mathrm{H}), 2.67(\mathrm{dt}, \mathrm{J}=$ 4.4. $12.5 \mathrm{~Hz}, 1 \mathrm{H}), 5.00(\mathrm{~s}, 1 \mathrm{H}), 5.08$ (pent, $\mathrm{J}=1.4,1 \mathrm{H}), 6.35(\mathrm{dd}, \mathrm{J}=5.2,2.8 \mathrm{~Hz}, 1 \mathrm{H})$; ${ }^{13} \mathrm{C}$ NMR $\left(67 \mathrm{MHz}, \mathrm{CDCl}_{3}\right) 9.18,18.43,19.30,27.48,28.73,30.36,85.47,114.85$, $123.72,134.48,143.30,172.73$.

Isobutyrate Ester 9b: Prepared and purified as propionate 9a to yield isobutyrate $9 \mathrm{~b}$ as a clear oil (5.425g, 80\%): IR (film) 2972, 1740, $1456 \mathrm{~cm}^{-1} ;{ }^{1} \mathrm{H}$ NMR $\left(270 \mathrm{MHz}, \mathrm{CDCl}_{3}\right)$ $1.18(\mathrm{~d}, \mathrm{~J}=6.9 \mathrm{~Hz}, 3 \mathrm{H}), 1.20(\mathrm{~J}=6.9 \mathrm{~Hz}, 3 \mathrm{H}), 1.60-1.72(\mathrm{~m}, 2 \mathrm{H}), 1.84(\mathrm{~s}, 3 \mathrm{H}), 1.87-1.93$ $(\mathrm{m}, 1 \mathrm{H}), 2.02-2.09(\mathrm{~m}, 1 \mathrm{H}), 2.18-2.31(\mathrm{~m}, 1 \mathrm{H}), 2.50-2.69(\mathrm{~m}, 2 \mathrm{H}), 5.00(\mathrm{~s}, 1 \mathrm{H}), 5.08$ (pent, $\mathrm{J}=1.4 \mathrm{~Hz}, 1 \mathrm{H}), 6.35(\mathrm{dd}, \mathrm{J}=5.3,2.8 \mathrm{~Hz}, 1 \mathrm{H}) ;{ }^{13} \mathrm{C} \mathrm{NMR}\left(67 \mathrm{MHz}, \mathrm{CDCl}_{3}\right)$ 18.47, 19.05 (intense), 19.31, 27.45, 30.29, 35.08, 85.15, 114.69, 123.27, 134.31, 143.39, 175.03 .

Isobutryate Ester 9c: Prepared and purified as propionate 9a except using 2-methyl-2cyclohexen-1-one to yield isobutyrate $9 \mathbf{c}$ as a clear oil (2.248 g, 52\%): IR (film) 2970 , $1733,1457 \mathrm{~cm}^{-1}$; ${ }^{1} \mathrm{H}$ NMR $\left(270 \mathrm{MHz}, \mathrm{CDCl}_{3}\right) 1.14(\mathrm{~d}, \mathrm{~J}=6.9 \mathrm{~Hz}, 3 \mathrm{H}), 1.15(\mathrm{~d}, \mathrm{~J}=6.9$ $\mathrm{Hz}, 3 \mathrm{H}), 1.49(\mathrm{~m}, 3 \mathrm{H}), 1.55-1.64(\mathrm{~m}, 2 \mathrm{H}), 1.69-1.76(\mathrm{~m}, 1 \mathrm{H}), 1.81(\mathrm{~s}, 3 \mathrm{H}), 1.90-1.97$ (m, $1 \mathrm{H}), 2.04-2.12(\mathrm{~m}, 1 \mathrm{H}), 2.42-2.56(\mathrm{~m}, 2 \mathrm{H}), 4.82(\mathrm{~s}, 1 \mathrm{H}), 4.97$ (pent, J = 1.6 Hz, 1H), 5.68 (m, $1 \mathrm{H}) ;{ }^{13} \mathrm{C}$ NMR $\left(67 \mathrm{MHz}, \mathrm{CDCl}_{3}\right) 18.53$ (intense), 19.07 (intense), 19.68, 24.96, 29.36, 35.07, 86.86, 113.61, 127.09, 132.99, 144.58, 175.15 .

Pentenoic Acid cis-11a: $n$-BuLi $(0.427 \mathrm{~mL}, 2.87 \mathrm{M}, 1.23 \mathrm{mmol})$ was added dropwise to a solution of diisopropylamine $(0.17 \mathrm{~mL}, 1.21 \mathrm{mmol})$ in ether $(10 \mathrm{~mL})$ at $-78^{\circ} \mathrm{C}$. After 15 min, isopropenyl propionate $9 \mathrm{a}(0.109 \mathrm{~g}, 0.40 \mathrm{mmol})$ in ether $(5 \mathrm{~mL})$ was added to the reaction mixture. After $2 \mathrm{~h}$ at $-78^{\circ} \mathrm{C}$, the mixture was slowly warmed to rt. The residue was isolated by extractive workup using $\mathrm{Et}_{2} \mathrm{O}$. The crude acid was dissolved in ether and treated with few drops of triethylamine. The mixture was purified by flash chromatography on silica gel using ether, followed by $1 \% \mathrm{AcOH} /$ Ether to give cis pentenoic acid 11a as a yellow oil (0.053 g, 49\%): IR (film) 2930, $1701 \mathrm{~cm}^{-1} ;{ }^{1} \mathrm{H}$ NMR $\left(270 \mathrm{MHz}, \mathrm{CDCl}_{3}\right) 1.14(\mathrm{~d}, \mathrm{~J}=6.7,3 \mathrm{H}), 1.63-1.76(\mathrm{~m}, 2 \mathrm{H}), 1.96(\mathrm{~s}, 3 \mathrm{H}), 2.17(\mathrm{dt}, \mathrm{J}=$ $4.2,6.5 \mathrm{~Hz}, 2 \mathrm{H}), 2.23-2.39(\mathrm{~m}, 3 \mathrm{H}), 2.51(\mathrm{dd}, \mathrm{J}=13.4,6.7 \mathrm{~Hz}, 1 \mathrm{H}), 2.58-2.69(\mathrm{~m}, 1 \mathrm{H})$, $6.10(\mathrm{t}, \mathrm{J}=4.2 \mathrm{~Hz}, 1 \mathrm{H}) ;{ }^{13} \mathrm{C} \mathrm{NMR}\left(67 \mathrm{MHz}, \mathrm{CDCl}_{3}\right) 16.41,21.89,23.19,28.42,29.64$, $38.49,39.18,118.85,131.70,131.78,134.53,182.50$. 
Pentenoic Acid cis-11b: Treatment of ester 9b in an identical fashion to 9a gave cis-acid 11b as a yellow oil (71\%): IR (film) 2932, $\left.1698 \mathrm{~cm}^{-1} ;{ }^{1} \mathrm{H} \mathrm{NMR} \mathrm{(270} \mathrm{MHz,} \mathrm{CDCl}_{3}\right) 1.20$ $(\mathrm{s}, 6 \mathrm{H}), 1.63-1.73(\mathrm{~m}, 2 \mathrm{H}), 1.95(\mathrm{~s}, 3 \mathrm{H}), 2.16(\mathrm{dt}, \mathrm{J}=4.2,6.5 \mathrm{~Hz}, 2 \mathrm{H}), 2.31-2.36(\mathrm{~m}, 2 \mathrm{H})$, $2.51(\mathrm{~s}, 2 \mathrm{H}), 6.08(\mathrm{t}, \mathrm{J}=4.2 \mathrm{~Hz}, 1 \mathrm{H}) ;{ }^{13} \mathrm{C} \mathrm{NMR}\left(67 \mathrm{MHz}, \mathrm{CDCl}_{3}\right) 23.14,24.03,25.30$ (intense), 28.35, 30.13, 43.24, 45.30, 119.12, 131.75, 133.04, 134.41, 185.12.

Pentenoic acids cis- and trans-11c: Treatment of ester 9c in an identical fashion to 9a gave a 5:1 mixture of acids trans-11c and cis-11c as a yellow oil (73\%):

cis-11c: - ${ }^{1} \mathrm{H}$ NMR (270 MHz, $\left.\mathrm{CDCl}_{3}\right) 1.15(\mathrm{~s}, 6 \mathrm{H}), 1.64(\mathrm{~m}, 2 \mathrm{H}), 1.70(\mathrm{~s}, 3 \mathrm{H}), 1.97$ (s, $3 \mathrm{H}), 2.08(\mathrm{~m}, 2 \mathrm{H}), 2.19(\mathrm{~m}, 2 \mathrm{H}), 2.66(\mathrm{~s}, 2 \mathrm{H}), 5.44(\mathrm{~m}, 1 \mathrm{H}) ;{ }^{13} \mathrm{C} \mathrm{NMR}\left(67 \mathrm{MHz}, \mathrm{CDCl}_{3}\right)$ 21.71, 23.78, 24.98, 25.22 (intense), 26.19, 29.68, 43.35, 45.51, 126.09, 19.07, 134.51, $136.59,185.79$.

trans-11c: - ${ }^{1} \mathrm{H}$ NMR $\left(270 \mathrm{MHz}, \mathrm{CDCl}_{3}\right) 1.19(\mathrm{~s}, 6 \mathrm{H}), 1.64(\mathrm{~m}, 2 \mathrm{H}), 1.79(\mathrm{~s}, 3 \mathrm{H}), 1.93(\mathrm{~s}$, $3 \mathrm{H}), 2.08(\mathrm{~m}, 2 \mathrm{H}), 2.19(\mathrm{~m}, 2 \mathrm{H}), 2.51(\mathrm{~s}, 2 \mathrm{H}), 5.48(\mathrm{~m}, 1 \mathrm{H}) ;{ }^{13} \mathrm{C} \mathrm{NMR}\left(67 \mathrm{MHz}, \mathrm{CDCl}_{3}\right)$ 23.54, 23.71, 24.86, 25.22 (intense), 26.33, 29.55, 43.29, 46.12, 125.69, 128.37, 135.23, $136.70,185.56$.

\footnotetext{
${ }^{1}$ Lindsay, H. A.;Salisbury, C. L.; Cordes, W.; McIntosh, M. C. Org. Lett. 2001, 3, $4007-$ 4010.

${ }^{2}$ Srikrishna, A; Vijaykumar, D. J. Chem. Soc. Perkin Trans I 2000, 2583-2589.
} 


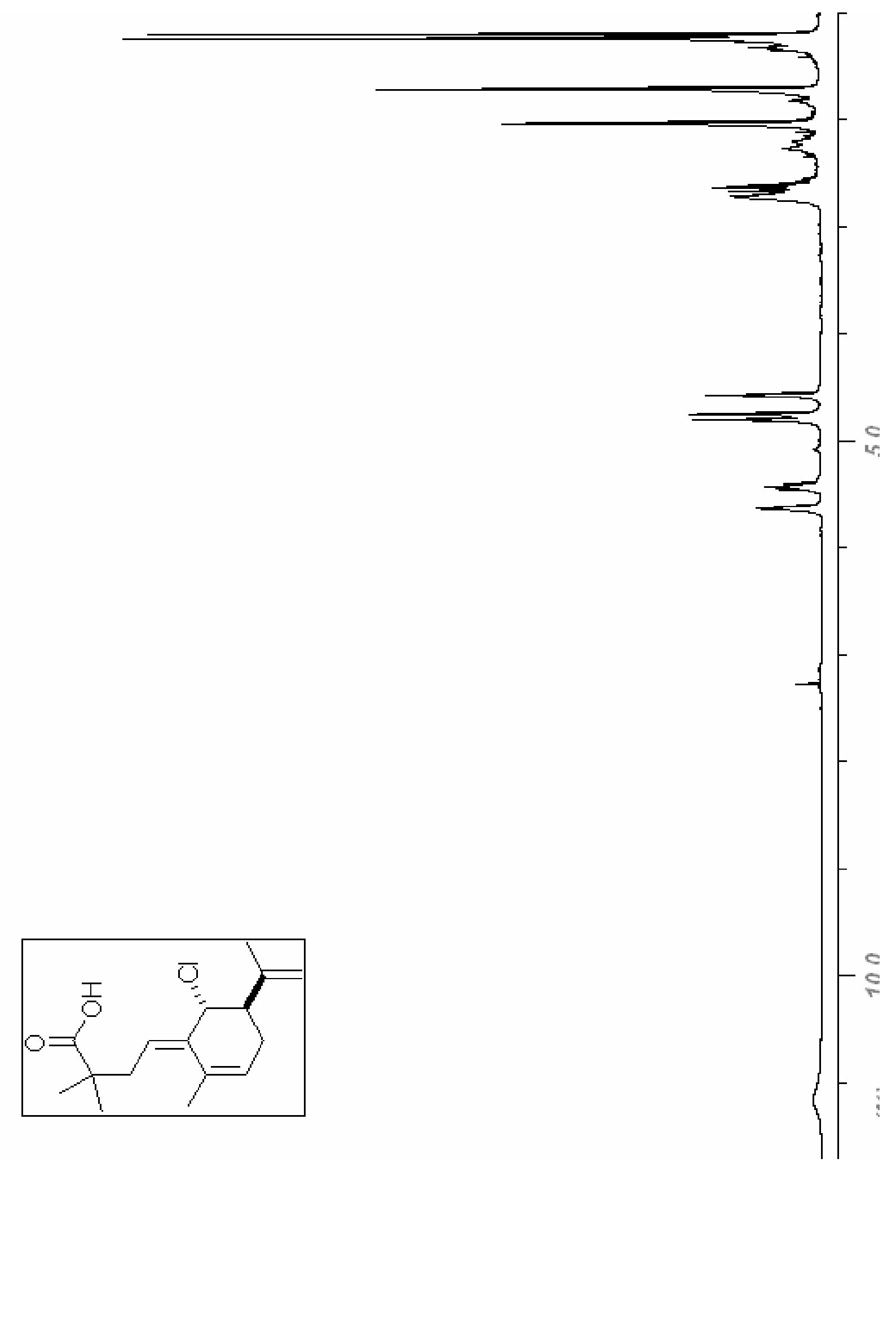




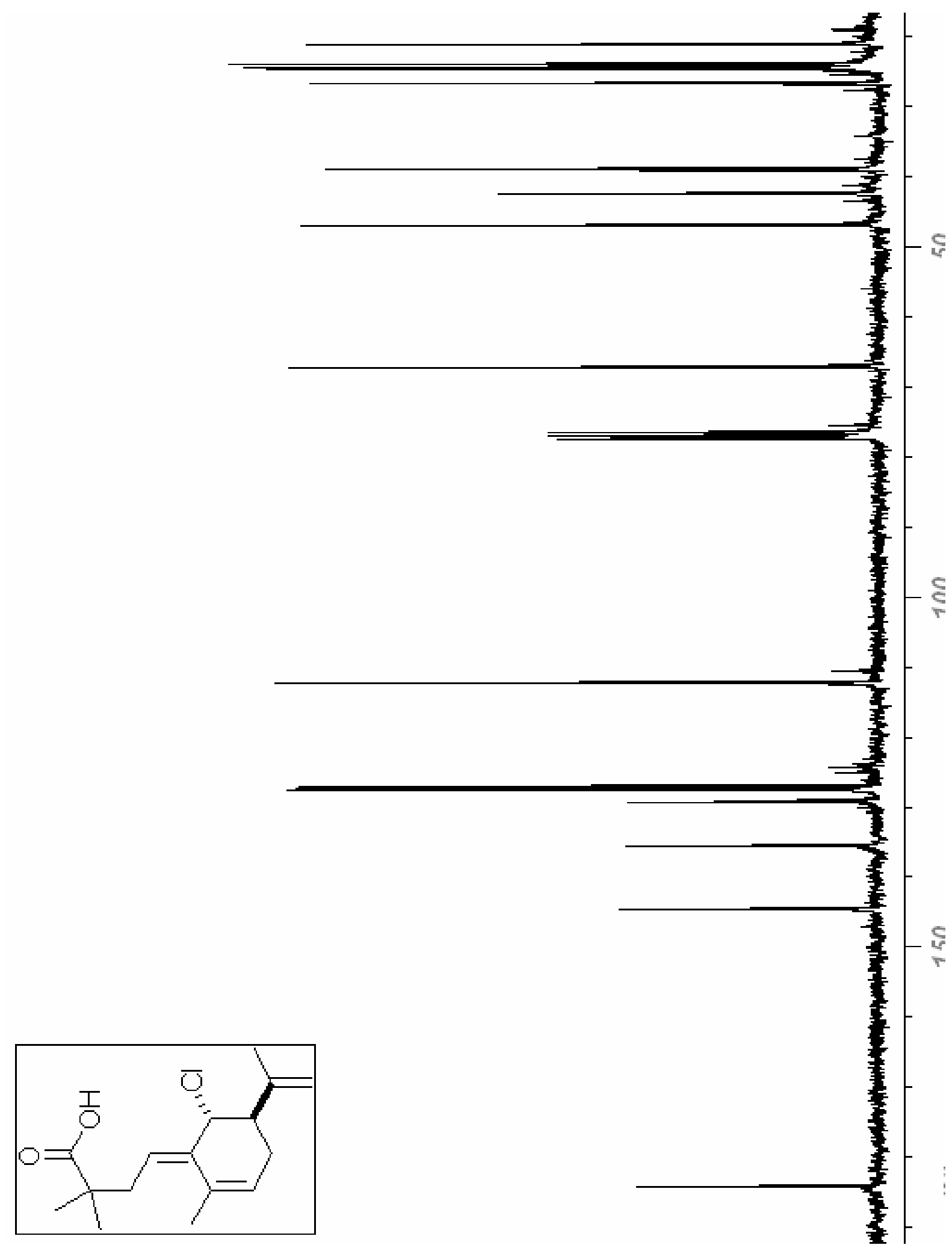




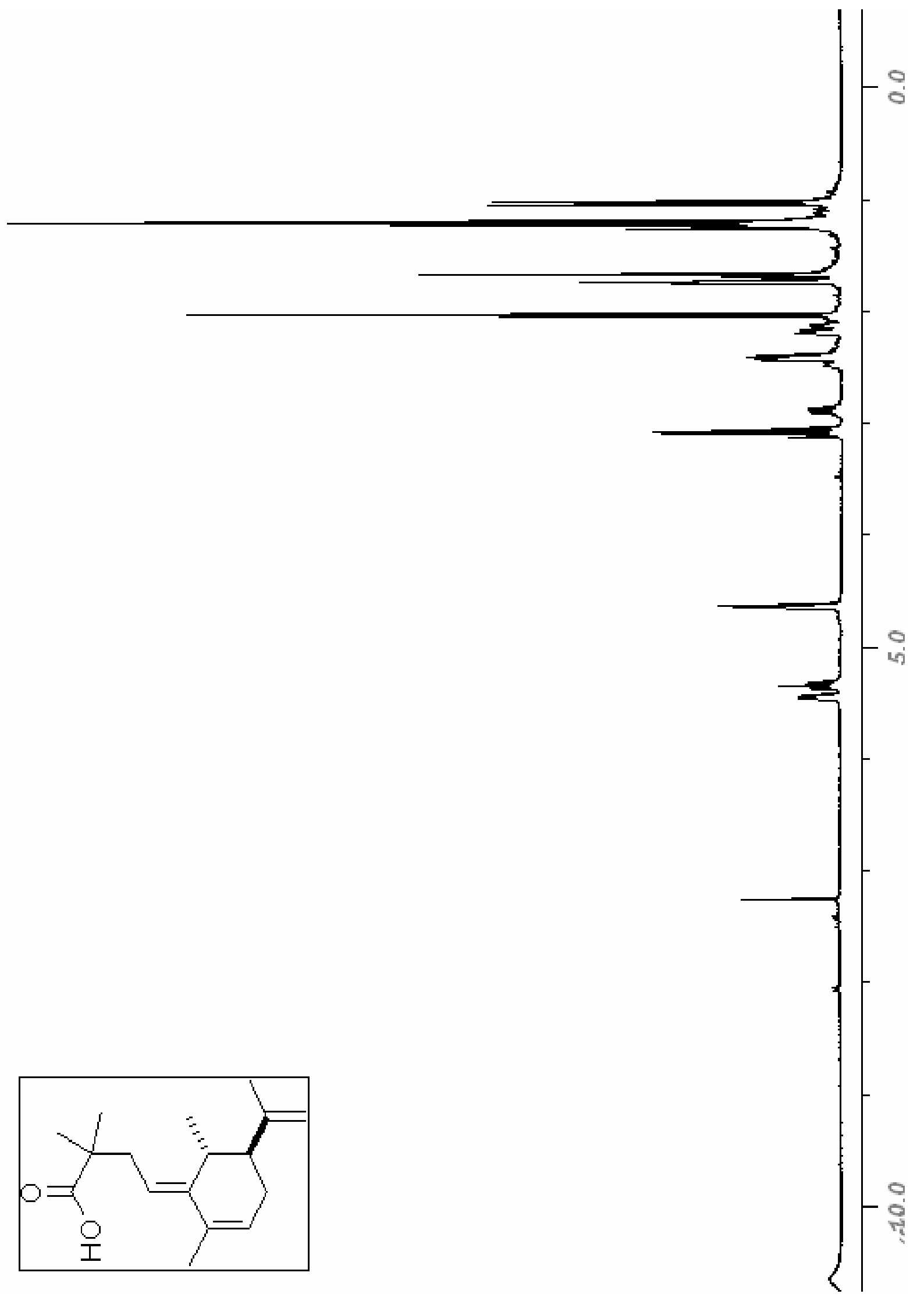




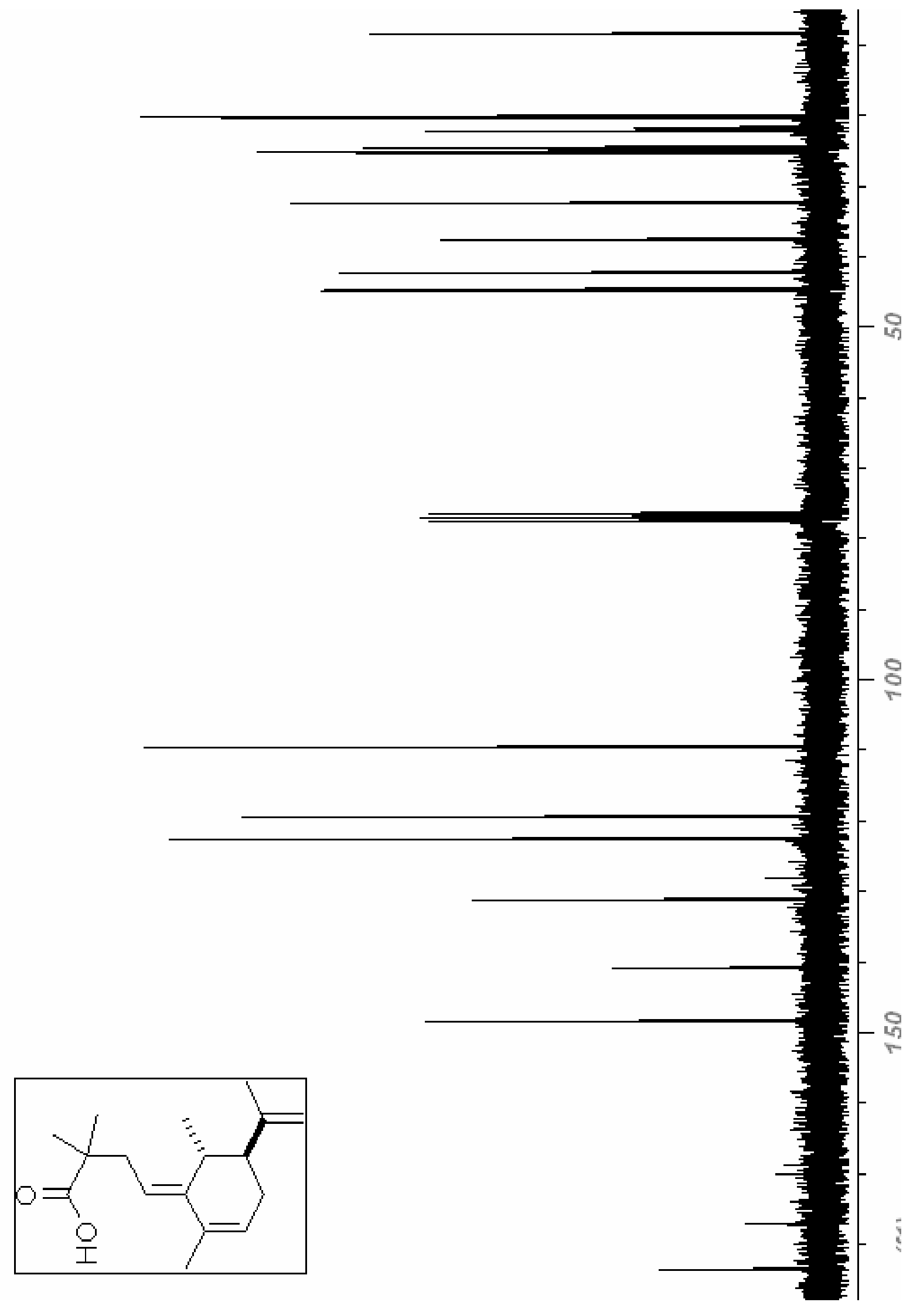

S10 


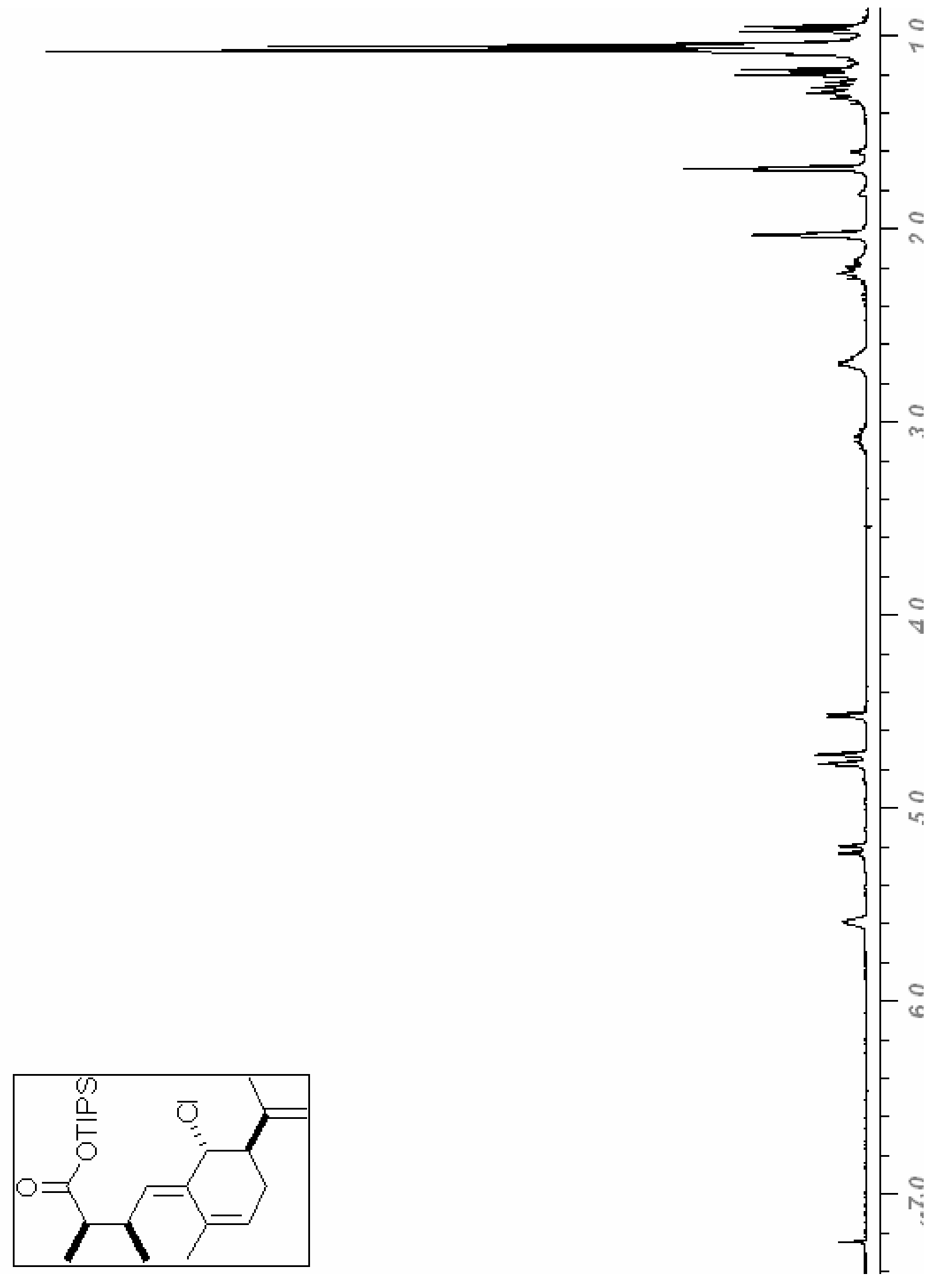




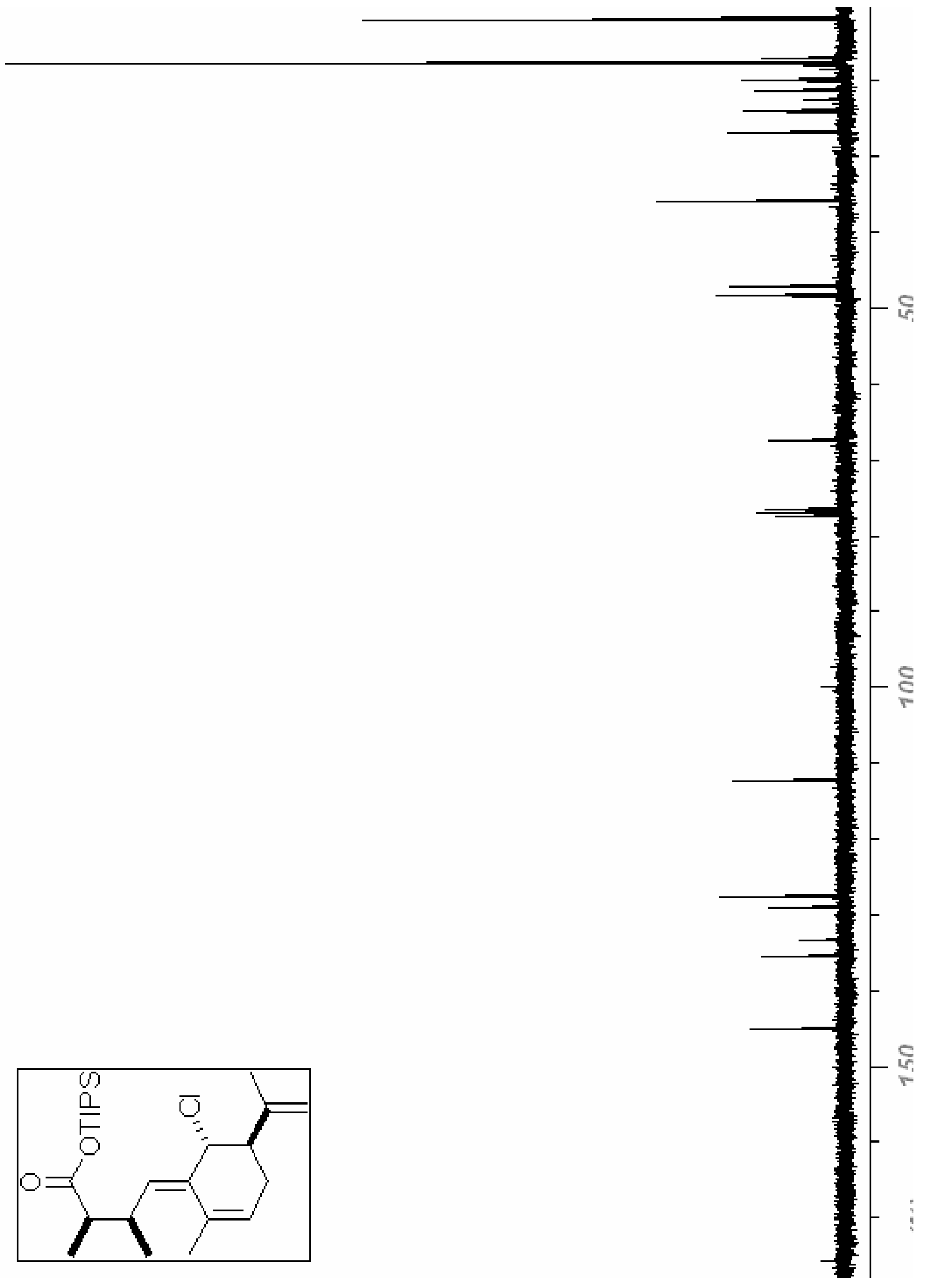

$\mathrm{S} 12$ 


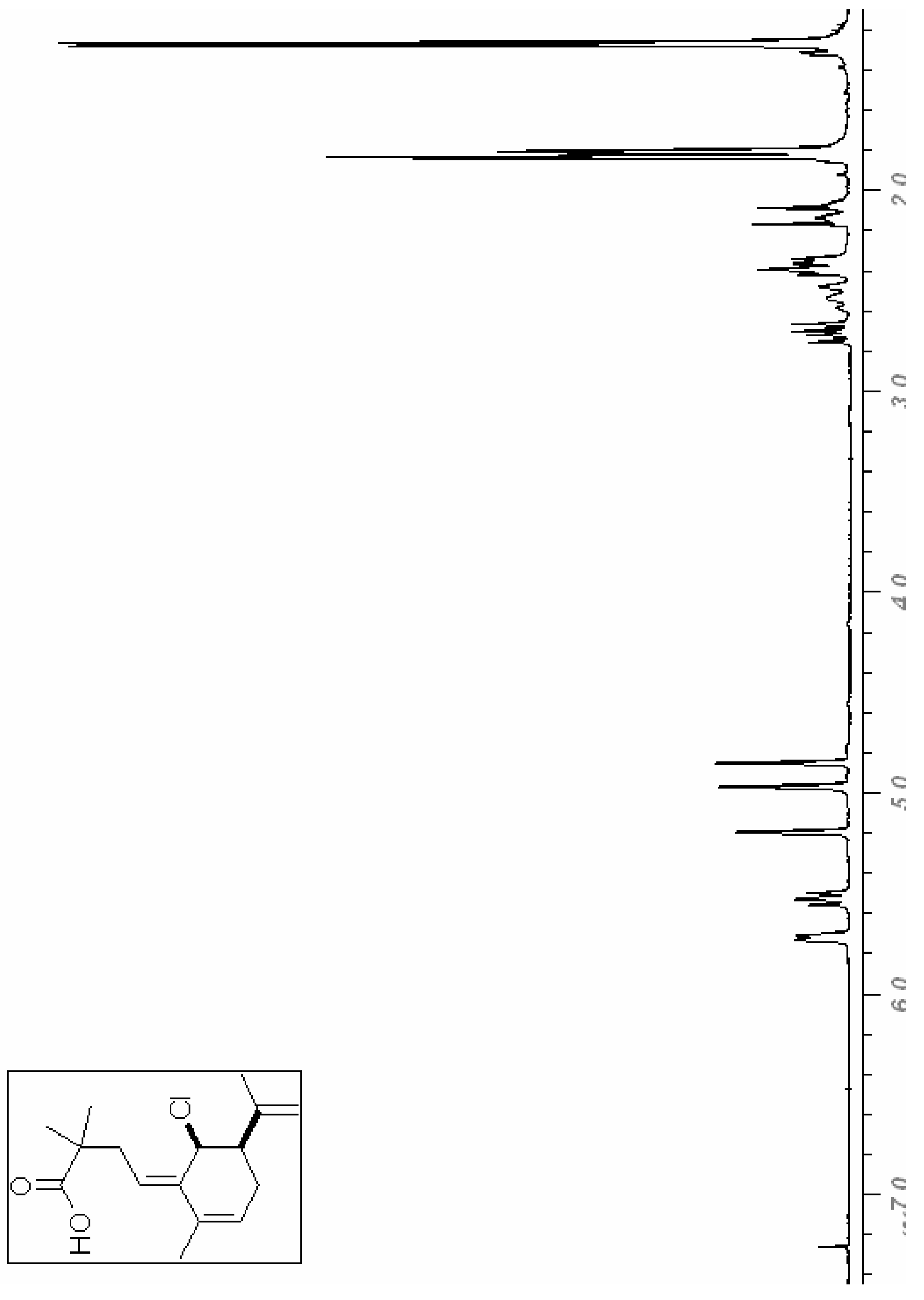




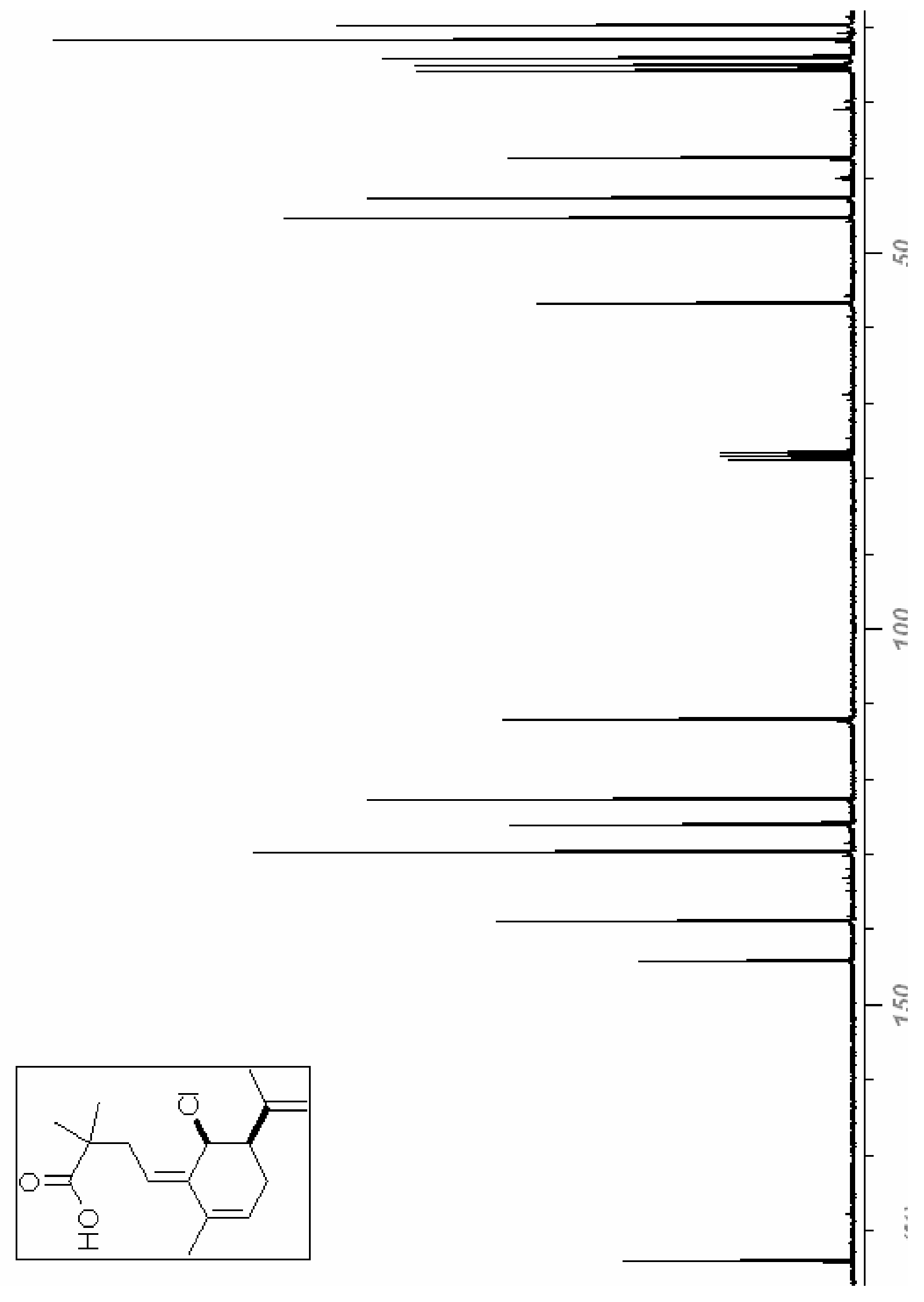

S14 


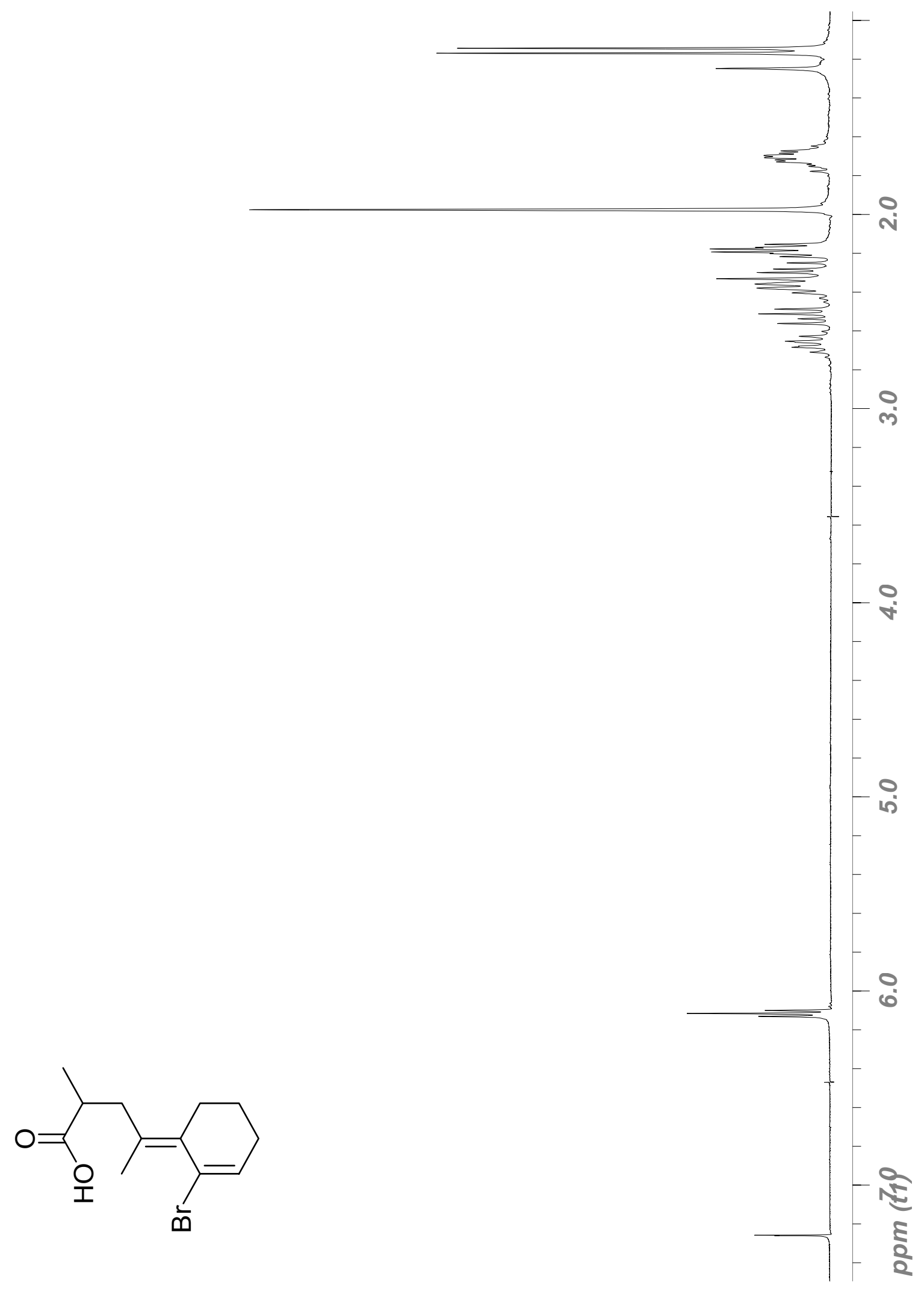




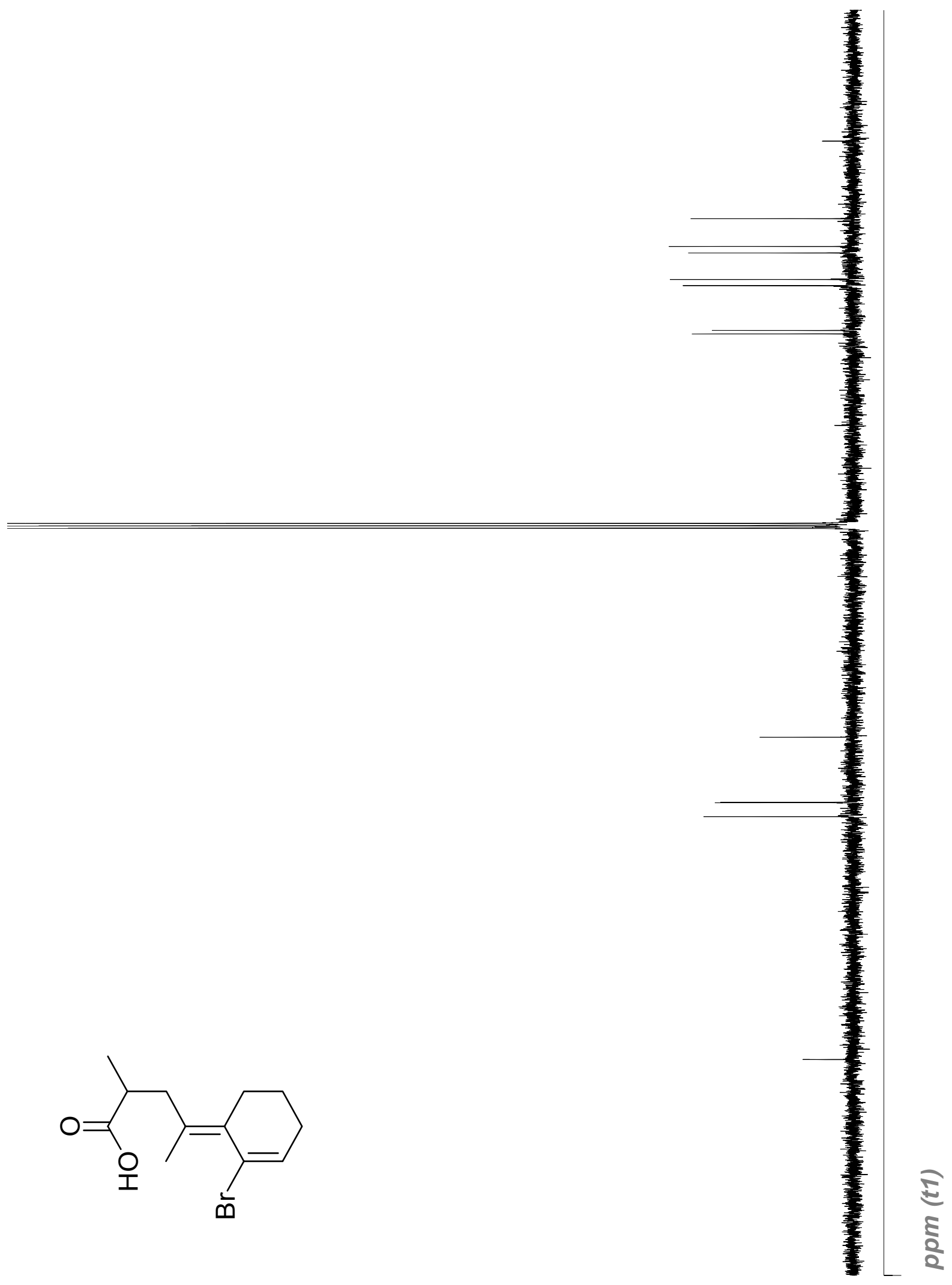




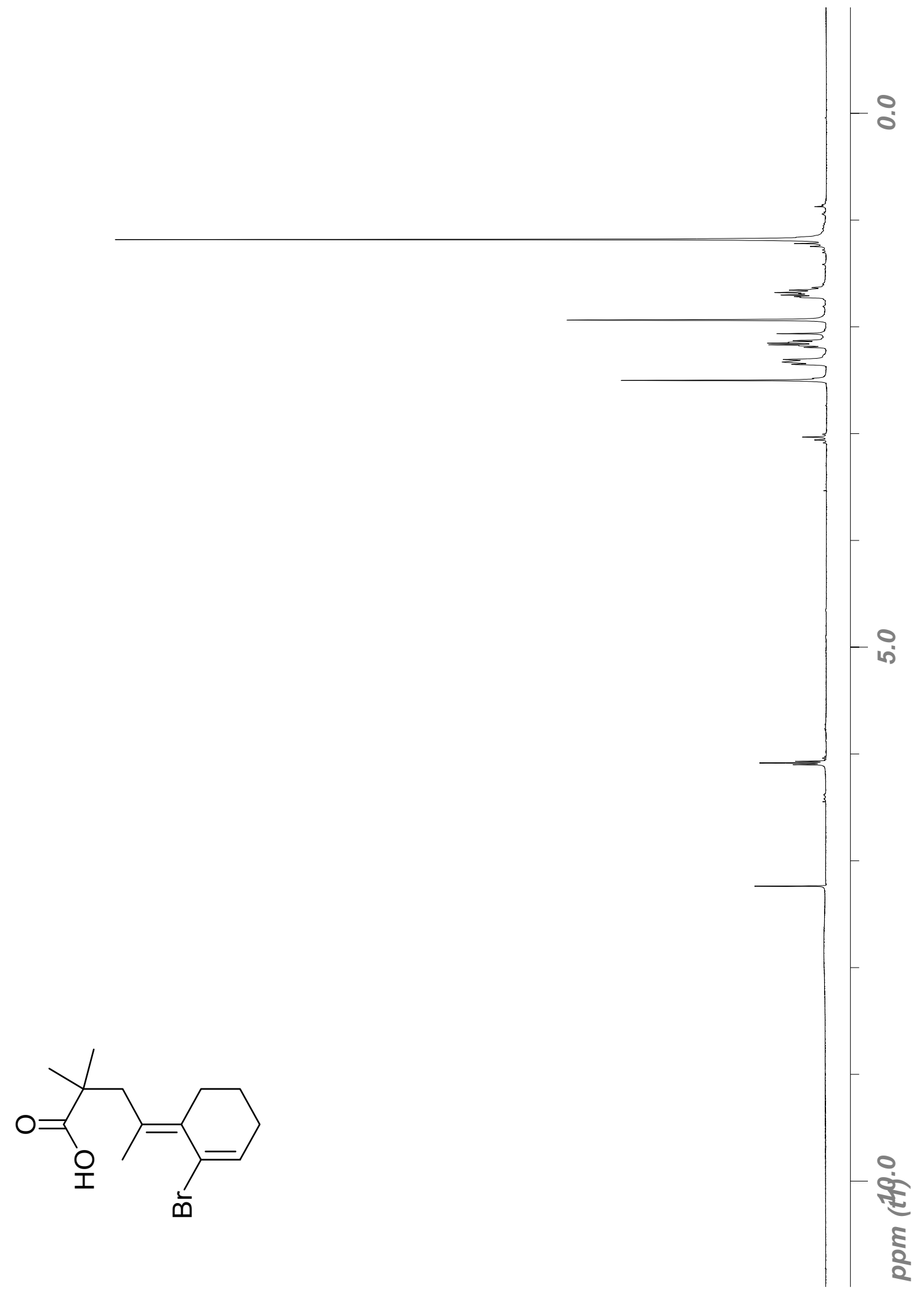




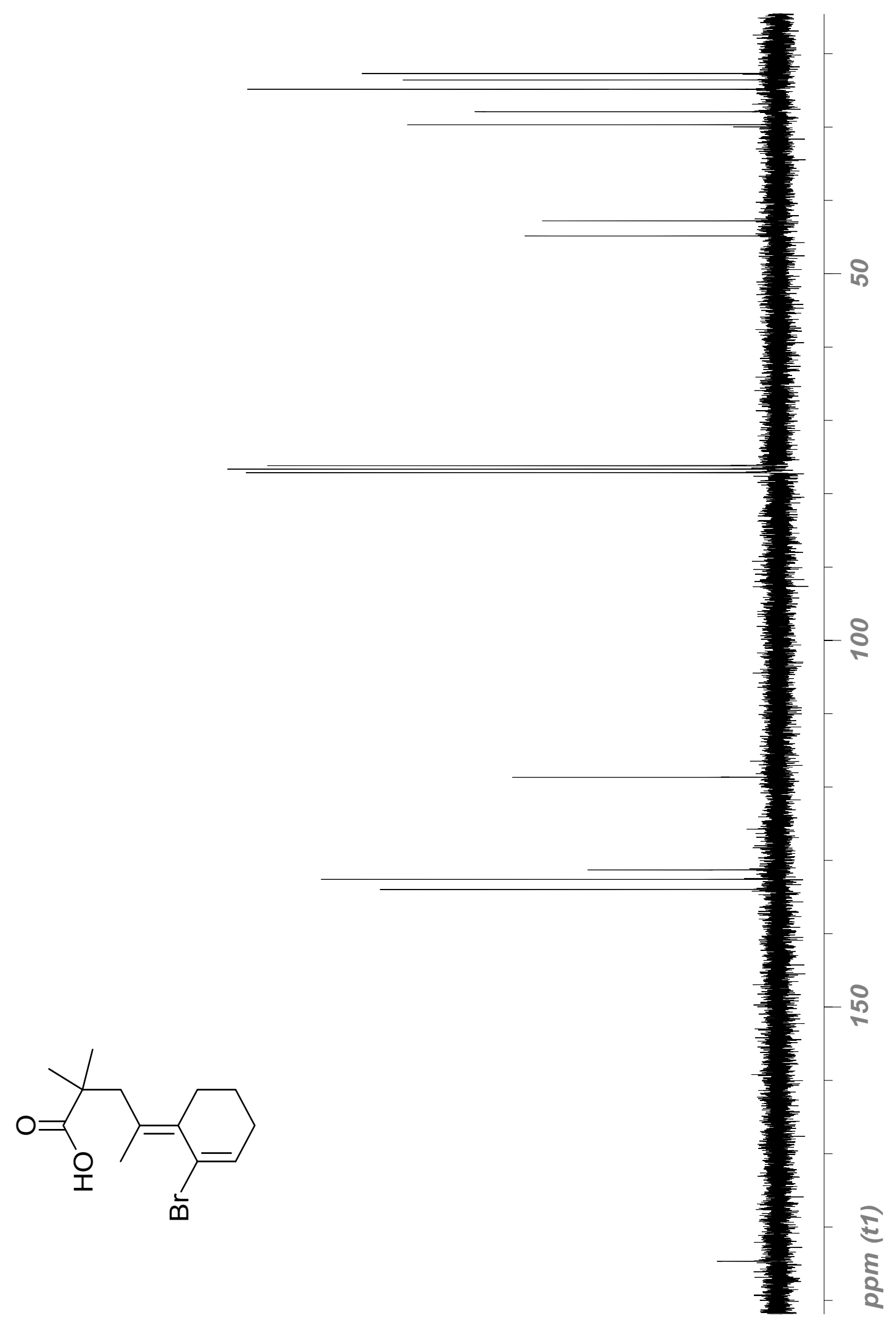




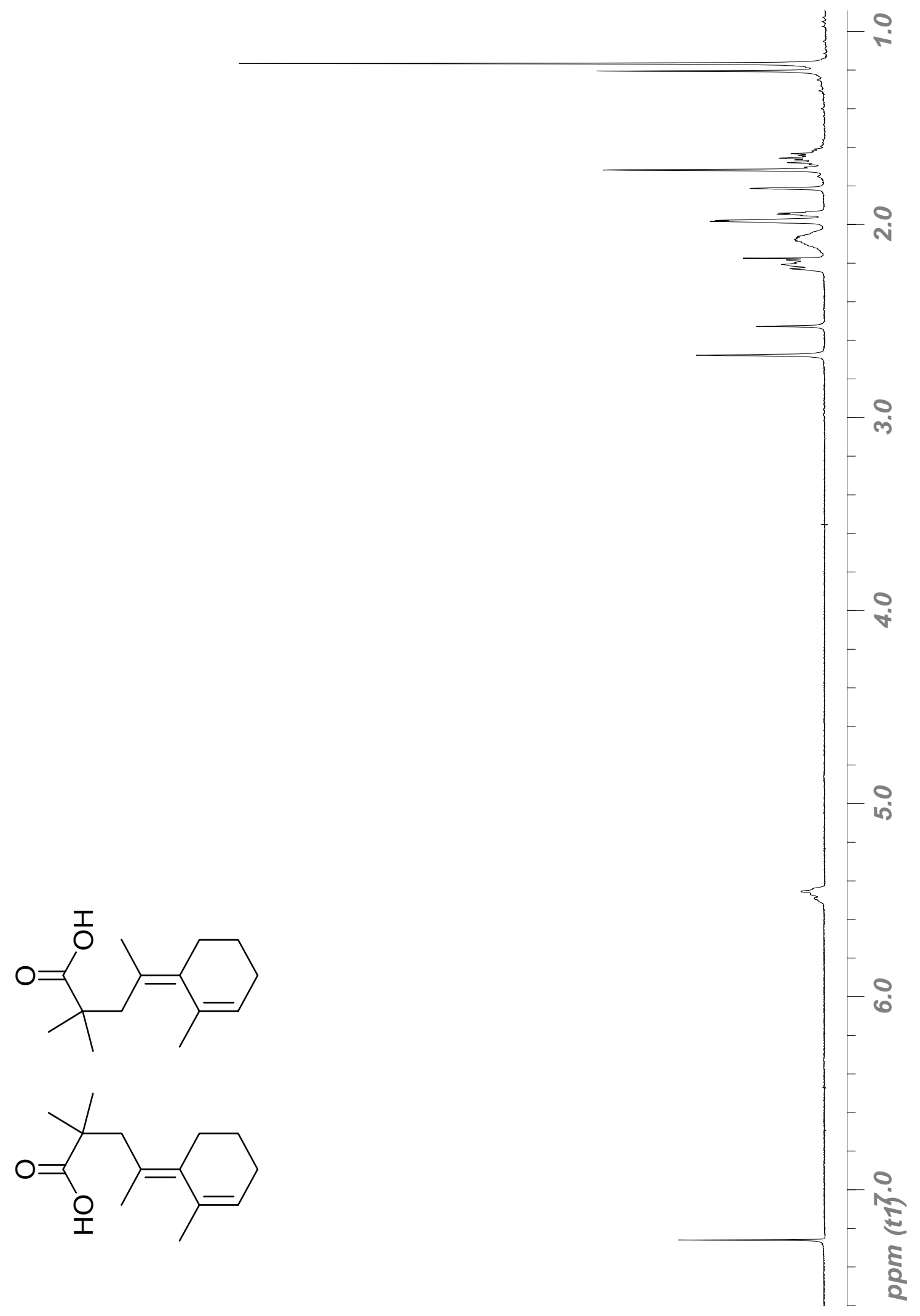




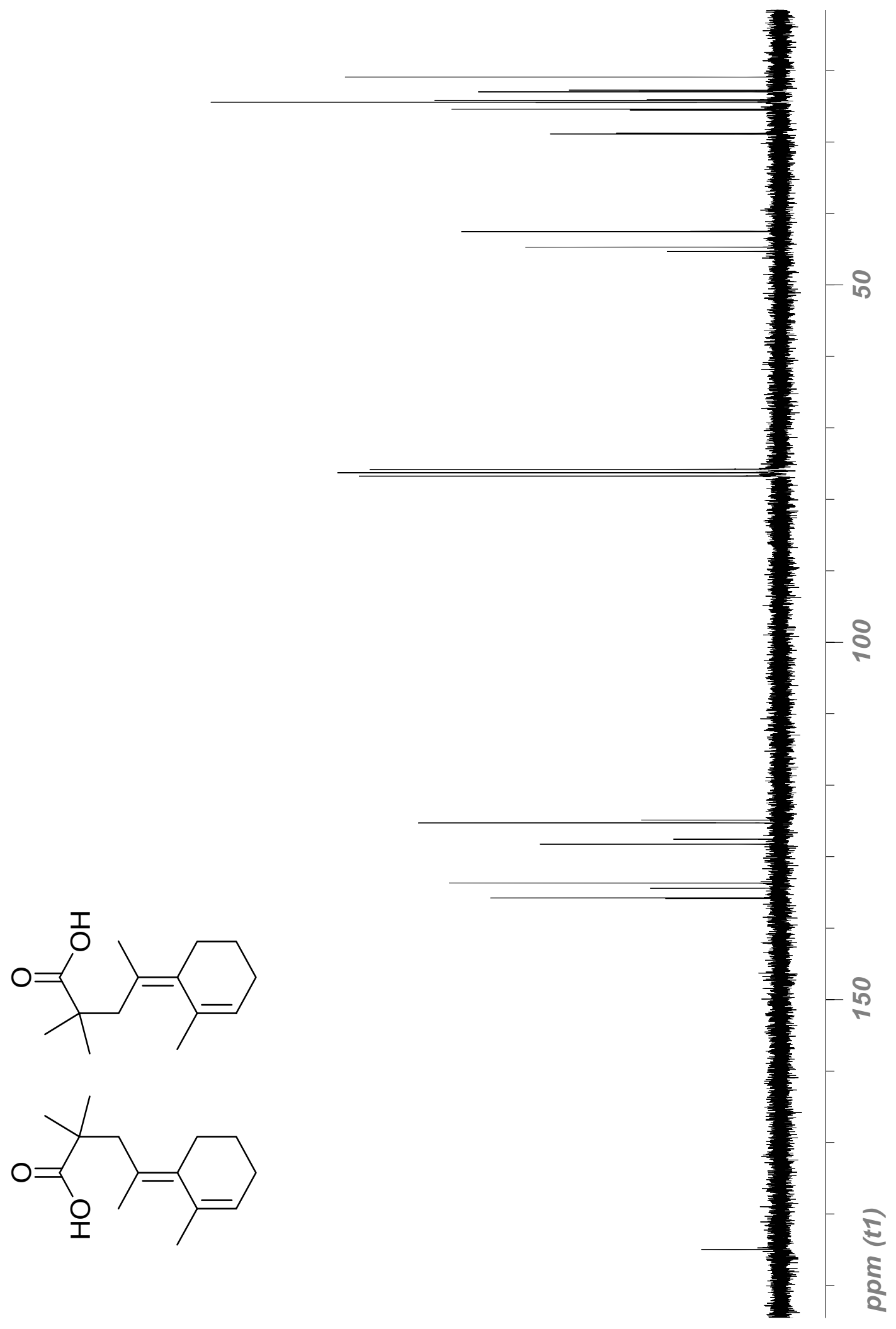

